University of Wollongong

Research Online

Faculty of Informatics - Papers (Archive)

Faculty of Engineering and Information

Sciences

June 2007

\title{
Use of Personal Digital Assistants (PDAs) in Medical Education
}

Rattiporn Luanrattana

University of Wollongong, r1631@uow.edu.au

Khin Than Win

University of Wollongong, win@uow.edu.au

John Fulcher

University of Wollongong, john@uow.edu.au

Follow this and additional works at: https://ro.uow.edu.au/infopapers

Part of the Physical Sciences and Mathematics Commons

\section{Recommended Citation}

Luanrattana, Rattiporn; Win, Khin Than; and Fulcher, John: Use of Personal Digital Assistants (PDAs) in Medical Education 2007.

https://ro.uow.edu.au/infopapers/651

Research Online is the open access institutional repository for the University of Wollongong. For further information contact the UOW Library: research-pubs@uow.edu.au 


\title{
Use of Personal Digital Assistants (PDAs) in Medical Education
}

\author{
Abstract \\ A systematic review of how the personal digital assistants (PDAs) have been used in healthcare \\ professions and medical education has been conducted in order to identify current usage of PDAs in both \\ areas. The major aim of this research is to study the feasibility of incorporating PDAs into problem-based \\ learning (PBL) medical education. A systematic review was conducted by exploring various databases on \\ the use of PDAs in two major areas: the healthcare professions and medical education during the period \\ 2000-2006. Results: The needs, patterns and functionalities of using PDAs in the medical profession have \\ been identified and categorized into different areas. What remains is to how to best incorporate PDAs into \\ a PBL approach to medical education at the University of Wollongong. \\ Disciplines \\ Physical Sciences and Mathematics

\section{Publication Details} \\ This conference paper was originally published as Luanrattana, R, Win, KT, Fulcher, J, Use of Personal \\ Digital Assistants (PDAs) in Medical Education, 20th IEEE International Symposium on Computer-Based \\ Medical Systems CBMS 2007, 20-22 Jun, 307-312.
}




\title{
Use of Personal Digital Assistants (PDAs) in Medical Education
}

\author{
Rattiporn Luanrattana, Khin Than Win, John Fulcher \\ Health Informatics Research Centre, University of Wollongong, Northfields Avenue, \\ NSW 2522, Australia \\ rl631@uow.edu.au
}

\begin{abstract}
A systematic review of how the personal digital assistants (PDAs) have been used in healthcare professions and medical education has been conducted in order to identify current usage of PDAs in both areas. The major aim of this research is to study the feasibility of incorporating PDAs into problem-based learning (PBL) medical education. A systematic review was conducted by exploring various databases on the use of PDAs in two major areas: the healthcare professions and medical education during the period 2000-2006. Results: The needs, patterns and functionalities of using PDAs in the medical profession have been identified and categorized into different areas. What remains is to how to best incorporate PDAs into a PBL approach to medical education at the University of Wollongong.
\end{abstract}

\section{Introduction}

Personal digital assistants (PDAs) are generic devices commonly used in both personal and professional spheres of society, due to their affordability and portability [1, 2]. In general, the aim of this research is to study the feasibility, applicability and functionalities of incorporating PDAs into a PBL approach to medical education. Hence, the research questions for this study are: (i) How PDAs and their medical software applications can be deployed in medical education and (ii) What are the key functionalities of PDAs applicable for a PBL curriculum medical education?

\section{Data sources}

Various key terms (handheld computers, handheld devices, PalmOS, PocketPC and Blackberry) were used to identify studies related to PDAs and their application in both the healthcare professions and medical education, from various databases (including Cinahl, Medline, Pubmed, Proquest 5000 and ScienceDirect). The journal article search was restricted to English-language articles published from 2000 to 2006.

The search strategy of using relevant keywords was used to gather journal articles and studies related to PDAs in healthcare, the medical professions and medical education. The article selection process began by screening the article's title, abstract, keywords and full-text that contained the related information. From 560 relevant journal articles, there were 140 articles (25\%) related to the use of PDAs in healthcare settings, and 96 articles (17\%) concerned with the use of PDAs in medical education. The articles were extracted and categorized into three major themes: (i) general characteristic of PDAs and platforms, (ii) PDAs in the medical professions, and (iii) how PDAs and their applications can be used in medical education. 


\section{General overview of PDAs}

PDAs can be used as personal information management (PIM) systems, data entry tools and storage devices [1,3], and other user-defined purposes [4]. Various ways for data to be entered are touch screen (using a stylus), attachable keyboard, and voice recognition. The voice recognition function helps users to enter the commands easily. Although PDAs have different approaches for data entry, there is no single data entry method that is appropriate for large amounts of data [3].

\subsection{PDA platforms}

PDAs utilise various types of operating system, each of which supports different types of information and synchronization protocols to a desktop computer. The three major operating systems (OS) are PalmOS, Microsoft and Symbian OS [1, 5]. Likewise, the major categories of PDAs are Palm, PocketPC and Smart phones of PDA phones [1]. The market share in 2004 for Palm, Microsoft PocketPC and Symbian was 14\%, 14\% and 65\%, respectively [5]. Smartphones that use Symbian OS are Nokia, Sony-Ericson and Motorola.

3.1.1 Palm OS or Pocket PC: Although there are various types of PDA in common usage, the majority is divided into two categories, based on the operating system (OS). The most popular one is PalmOS, which is small in size and easy to use. A lot of application software is available for PalmOS based-systems [6-8]. Another reason why PalmOS became the most popular is that it is less expensive than the PocketPC. PocketPC is another type of PDA that uses Microsoft Windows as its operating system. PocketPC requires more memory space and faster processor speed. This is the reason why the price of PocketPCs is higher than PalmOS. The PocketPC is less popular than PalmOS because of more limited software availability. Another drawback of PocketPC is its slow operating speed, as it requires more processing to finish a simple task [9]; it also has a shorter battery life than PalmOS [7]. PalmOS-based systems, therefore, have become more popular in medicine [7, 9]

3.1.2 PDA phones SymbianOS: There is another type of PDA, which is called "Smartphone" or "PDA phone". The Smartphone OS is different from other PDAs that use PalmOS or Microsoft Windows for PocketPCs - namely SymbianOS.

\section{Symbian OS}

Symbian OS is also known as EPCO, (which was created for Psion Series 5 PDA). The OS is stored on flash or read-only memory (ROM), and can be upgraded at some future time. The drawback of Symbian is maintaining code compatibility with other platforms. This is a major reason why third party developers often experience steep learning curves [5].

\section{Design of Symbian}

One good aspect of the Symbian OS design is that each software application can run its own protection process. This means it is unable to access other part of memory. The strength of Symbian is that it cannot execute code of other devices. On the other hand, software installation packages can be installed via PC suite by retrieving and opening from attachments [5].

\section{PDAs in the healthcare professions}

To effectively the use PDAs in a healthcare organization, they need to be integrated into the existing organization systems and connected into the network for communication and data sharing [4]. The three major advantages of incorporating PDAs into healthcare 
organizations, particularly in busy units, are: 1) to deliver essential clinical information to physicians and healthcare staff wherever and whenever they need, 2) physicians being able to order any medical test at their finger tips, and 3) the use of PDAs provides the opportunity for physicians to record their medical notes or create referral letters by using the voice recording function within the PDA [4].

\subsection{Why are PDAs used in medical and nursing professions?}

There are a few major reasons why PDAs are used in both the medical and nursing professions. Firstly, PDAs provide speed for data collection and data analysis [10]. Secondly, PDAs are widely used in both medical and nursing practices. Thirdly, PDAs are becoming widely used for educational purposes [11-14].

\subsection{Patterns of using PDAs in medical practices}

The pattern of using PDAs in medicine depends on various factors, including functionalities, PDA platform, basic functionalities, data security and privacy, limitation of PDAs and PDA functionalities for medical use [15, 16]. However, the major factor of PDA usage in medical practice is relatively based on the available functionalities that support medical use [2].

It appears that the pattern of using PDAs in medical practices depends on various factors, including age, gender, location of healthcare providers and status of users, where the users' status can be defined as students and medical residents, general practitioners and specialists [2]. Younger physicians seem to use PDAs more than older physicians [2].

Also, younger medical students and residents are more likely to use PDAs for their medical study on a daily basis [17]. Several studies further indicate that male physicians are more likely to use PDAs than female physicians [2, 18, 19].

Location of healthcare providers also has an impact for pattern on using PDAs: physicians who work far away from a major city or in an urban area seem to use PDAs more often than physicians in city $[2,19,20]$.

The needs of PDA users in the healthcare professions can be categorized into administrative tasks and direct clinical work [18-22]. The PDA functionalities that general practitioners and specialists normally used are word processing, calculator, charge capture, patient tracking, resident hours, telephone message, general time management, personal information management (PIM), patient referrals, procurement of supplies, patient census, order entry, dictation, billing and coding [2].

\subsection{The functions of PDAs in the medical professions and clinical practice}

The PDA has become a popular device in different fields of work especially in healthcare, clinical area and also in medical education [23]. Most physicians use PDAs at the point of care to access drug and clinical information [18, 19], for clinical decision support, for prescribing medication and for viewing lab results wirelessly [19, 21]. Frequently used software applications in the medical professions are pharmacopoeias, medical references and clinical calculators [22]. Clinicians use PDAs to gather, enter and store data at the point-ofcare, after which the data can be downloaded to a central computer. Clinicians also use PDAs for recording patient information, patient logs, clinical hours, clinical reports and clinical references [18]. Therefore using PDAs in clinical practice can assist doctors and clinical residents to access and manage information [24]. 
PDAs are generally used for five major functions in healthcare networks, these being: general software applications, referencing, organisation, communication and other special software applications.

\section{How can PDAs and their applications in medicine be deployed in medical education?}

With the basic understanding of PDA usage patterns and major PDA functionalities in healthcare networks and clinical practice, it is necessary to consider the trend of using PDA applications in such areas. The use of PDAs in the medical professions is gradually increasing due to ever reducing training and hardware costs. PDA usage in the medical professions is moving towards PIM, drug referencing and online accessing [2].

In addition, the use of PDAs will increase as younger medical professionals start their training, and choose to use the devices. This is proving true as medical schools are introducing PDA technology into medical education and residency programs $[12,14,15,18$, 19, 22, 25-27].

According to the literature, with regard to the use of PDAs in medical education, numerous medical schools have already used PDAs in the medical education such as George Washington University, Thomas Jefferson Medical College, Georgetown University, Columbia University, Stanford University and Wayne State University. While not many medical schools have yet combined PDAs into medical curricular, as have Johns Hopkins University and the Medical College of Wisconsin, say -but they do have future plans to include PDAs. The purpose of using PDAs in medical education are to keep track with student- and patient-logs, review medical articles, take tests via PDAs, keep relevant medical references (such as drug databases, core medical textbooks), keep track of class assessment and classroom evaluation.

The advantages of incorporating PDAs into medical education are to provide the alternative ways of learning, taking notes, and updating relevant information with regard to their learning, as well as reducing medical errors. Moreover, students can have other ways of communication while they are in both clinical placement and on the campus.

It is unnecessary for students to rely on desktop or laptop computers, as PDA functionalities are mostly identical, they also have network accessibility. Other advantages are cost and time savings in term of manpower and hardware-software installation.

However, there are also drawbacks in using PDAs in medical education. In terms of technical aspects, problems may occur with software installation and loss of data due to improper backup, particularly with the use of student- and patient-log systems [26]. Other problems are: technology compatibity, data security, scalability and data synchronization [27].

Various PDA applications can be used in medical education, including student- and patient-log systems, classroom assessment systems, teaching and interaction evaluation, objective structured clinical examinations (OSCE), references applications for drug interactions and e-medical textbooks. The aforementioned applications refer to medical education generally, and not to the specific needs of PBL medical curricula.

In order to determine these needs, the research design has been setout with both interview and focus group of both medical and IT experts from the Graduate Medical School (GSM), University of Wollongong in order to validate the findings against the literature review. The several interview questions with regard to the use of PDA applications in medicine, clinical practices and medical education will be asked. For instance: What sort of information / other PDA software applications in medicine do you believe would be beneficial for the students to study medicine in PBL approach?, If PDAs are to be used in PBL curricula, how would you 
encourage the students to understand, use PDA technology, be efficient and accurate in information gathering, in order to optimize and improve their medical studies?

After interviews have completed, the data will be analyzed and interpreted in order to formulate the focus group questions in the next phase of this study.

\section{Conclusion}

In general, the advantages of using PDAs in medical education are to provide alternative ways of learning, to consult medical references, to take tests, and to update relevant information; while the drawbacks include difficulties in software installation, and loss of data due to improper backup, technology compatability, data security, scalability and data synchronization. What have been found particularly useful for PBL medical curricula at the University of Wollongong are clinical and student-log -future investigation will confirm whether or not this is the case.

\section{Acknowledgements}

The authors would like to gratefully acknowledge all assistance of Prof. Don Iverson and Prof. Patrick Crookes, and Assumption University for scholarship support.

\section{References}

[1] W. Jansen and R. Ayers, "An overview and analysis of PDA forensic tools," Digital Investigation, vol. 2, pp. 120-132, 2005.

[2] C. Garritty and K.E. Emam, "Who's Using PDAs? Estimates of PDA Use by Health Care Providers: A Systematic Review of Surveys," Journal of Medical Internet Research, vol. 8, 2006.

[3] R.S. Laskin and J. Davis, "The Use of a Personal Digital Assistant in Orthopaedic Surgical Practic," From The Knee Service Hospital for Special Surgery, New York, NY, New York, Report April 2004.

[4] P. Turner, G. Milne, M. Kubitscheck, I. Penman, and S. Turner, "Implementing a wireless network of PDAs in a hospital setting," Personal and Ubiquitous Computing. , vol. 9, pp. 209-217, 2005.

[5] G. James, "Malicious threats to Smartphones," Network Security, vol. 2004, pp. 5-7, 2004.

[6] J.J. Choi, "Teaching with a Personal Digital Assistant," Journal of the American College of Radiology, vol. 2, pp. 825-832, 2005.

[7] D.F. Naylor, "The PDA saga continues: updates and essential and nonessential gadgets," Current Surgery vol. 59, pp. 402-403, 2002.

[8] A. Jahan, B. Gretter, and M.P. Smith, "The anesthesiologist's guide to personal digital assistants," Regional Anesthesia and Pain Medicine vol. 27, pp. 193-196, 2002.

[9] Anonymous, "PDAs in Family Medicine Practice," vol. 2006: University of Rochester Medical Center, 2005c.

[10] C. Jao, D.B. Hier, and J. Su, "Evaluating a Digital Resident Diagnosis Log: Reasons for Limited Acceptance of a PDA Solution," presented at Proceeding of AMIA2003 Symposium, Washington, DC, 2003.

[11] Anonymous, "Epocrates Goes Back to School," in Health Data Management, vol. 13. New York, 2005b, pp. 34.

[12] B. Lopez, P.F. Kolecki, D.F. Louis, and C. Ravinowitz, "The use of a personal digital assistant Patient Encounter Log System to track procedures performed by students during a mandatory emergency medicine clerkship," Annals of Emergency Medicine, vol. 44, pp. 48-49, 2004. 
[13] D.R. Masys, P.F. Brennan, J.G. Ozbolt, M. Corn, and E.H. Shortliffe, "Are Medical Informatics and Nursing Informatics Distinct Disciplines?: The 1999 ACMI Debate.," Journal of American Medical Informatics Association, vol. 7, pp. 304-312, 2000.

[14] B.E. Mays and B.J. Boston, "PDAs @ the library. Introducing PDAs in preclinical medical education," Journal of Electronic Resources in Medical Libraries, vol. 1, pp. 85-91, 2004.

[15] S. Fischer, T.E. Stewart, S. Mehta, R. Wax, and S.E. Lapinsky, "Handheld Computing in Medicine," Journal of American Medical Informatics Association, vol. 10, pp. 139-149, 2003.

[16] D.C. Baumgart, "Personal digital assistants in health care: experienced clinicians in the palm of your hand?," The Lancet, vol. 366, pp. 1210-1222, 2005.

[17] D.F. Criswell and M.L. Parchman, "Handheld Computer Use in U.S. Family Practice Residency Programs," Journal of American Medical Informatics Association, vol. 9, pp. 80-86, 2002.

[18] S.D. Stroud, E.A. Erkel, and C.A. Smith, "The use of personal digital assistants by nurse practitioner students and faculty," Journal of the American Academy of Nurse Practitioners, vol. 17, pp. 67-75, 2005.

[19] A.E. Carroll and D.A. Christakis, "Paediatricians' Use of and Attitudes About Personal Digital Assistants," Pediatrics, vol. 113, pp. 238-242, 2004.

[20] A.E. Carroll, P. Tarczy-Hornoch, E. O'Reilly, and D.A. Christakis, "The effect of point-of-care personal digital assistant use on resident documentation discrepancies," Pediatrics vol. 113, pp. 450-454, 2004.

[21] M. Larkin, "Handheld use increasing for e-learning and clinical decision making," The Lancet, vol. 361, pp. 93, 2003.

[22] A. Jotkowitz, J. Oh, C. Tu, D. Elkin, L.A. Pollack, and H. Kerpen, "The use of personal digital assistants among medical residents," Medical Teacher, vol. 28, pp. 382-384, 2006.

[23] R.C. Alvarez, "The promise of e-Health - a Canadian perspective," EHealth International The Journal of Applied Health Technology, vol. 1, 2002.

[24] M. Al-Ubaydli, "Handheld computers," British Medical Journal, vol. 328, pp. 1181-1184, 2004.

[25] P. Wilson, R. Billingsley, and L. Pellegrino, "PDA power at the bedside," Medical Reference Services Quarterly, vol. 24, pp. 1-7, 2005.

[26] R.J. Kurth, V. Silenzio, and M.M. Irigoyen, "Use of personal digital assistants to enhance educational evaluation in a primary care clerkship," Medical Teacher, vol. 24, pp. 488-490, 2002.

[27] A.S. Menon, S. Moffett, M. Enriquez, M.M. Martinez, P. Dev, and T. Grappone, "Audience Response Made Easy: Using Personal Digital Assistants as a Classroom Polling Tool," Journal of American Medical Informatics Association, vol. 11, pp. 217-220, 2004. 